\title{
In-Line Series-Feed Collinear Slot Array Fed by a Coplanar Waveguide
}

\author{
Shih-Yuan Chen, I-Ching Lan, and Powen Hsu
}

\begin{abstract}
The in-line series-feed collinear slot array antenna fed by a coplanar waveguide is investigated thoroughly. Radiating elements are placed end-to-end along the shunt slotline pair transverse to the feedline. This simple linear array structure increases the antenna gain and shapes the H-plane pattern. The shunt slotline pair can be terminated by either short or open circuit. The short ends, the slotline circular open stubs, and the directly-truncated open ends are used as terminations. Design guidelines for the three cases are given separately. A reflector-backed arrangement, with which the proposed antennas would be able to radiate unidirectional patterns, is also discussed. Experimental results, which agree well with the simulated ones, verify the performances of the proposed antennas.
\end{abstract}

Index Terms-Coplanar waveguides, linear arrays, slot antennas, slot arrays.

\section{INTRODUCTION}

$\mathbf{D}^{-1}$ UE TO THE low profile, light weight, and high directivity, planar antenna arrays have been employed in many microwave and millimeter-wave applications, such as the access points of the wireless local area networks (WLAN) and the base stations of the point-to-multipoint fixed broadband wireless access (BWA) systems based on the IEEE standard 802.16 wireless metropolitan area networks (WirelessMAN) [1]. Most of the planar antenna arrays have been designed by using the microstrip-line feeds, however, relatively fewer works so far have used the coplanar waveguide (CPW) as feedlines [2]-[9]. The $\mathrm{CPW}$ has gained more and more popularity since it has many advantages over the microstrip-line, such as the lower radiation loss, less dispersion, and easier integration with the active and passive devices. Moreover, using apertures as radiating elements in the CPW-fed planar antenna arrays will make the structure uniplanar and facilitate the input impedance matching [5]. Thus, in this paper, we propose a novel in-line series-feed collinear slot array antenna fed by a CPW. The basic concept of this antenna is quite similar to that of the wire dipole antenna first proposed by C. S. Franklin in 1925 [10]-[13]. Three possible designs of the newly proposed antenna with the shunt slotline pair terminated separately by the short ends, the slotline circular open stubs, and the truncated open ends are discussed. A simple method, which

Manuscript received September 22, 2006; revised February 27, 2007. This work was supported by the National Science Council, Taiwan, R.O.C., under Contract NSC 95-2752-E-002-002-PAE.

The authors are with the Department of Electrical Engineering and the Graduate Institute of Communication Engineering, National Taiwan University, Taipei 10617, Taiwan, R.O.C. (e-mail: sychen@cc.ee.ntu.edu.tw; phsu@cc.ee.ntu.edu.tw).

Digital Object Identifier 10.1109/TAP.2007.898620

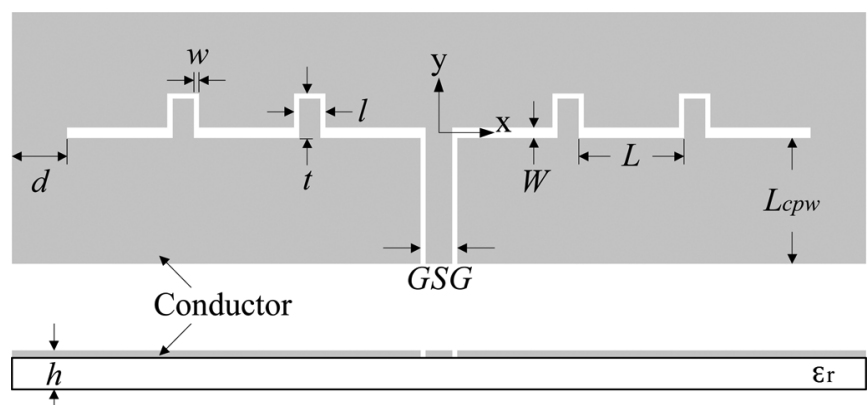

Fig. 1. Geometry of six-element in-line series-feed collinear slot array fed by a CPW.

is employed to make the radiation patterns of the proposed antennas unidirectional, is described. Prototype antennas are built and their performances are measured extensively. Experimental results for input return losses, radiation patterns, and in-band peak gains are presented. Comparisons among the performances of the proposed antennas are also presented.

\section{ANTENNA DESIGN}

\section{A. Short-Ended Case}

The geometry of the proposed in-line series-feed collinear slot array antenna fed by a CPW is depicted in Fig. 1. This antenna has a simple structure with only one layer of dielectric substrate and metallization. A pair of shunt slotlines, along which the horizontal end-to-end slotline sections of length $L$ are separated and interconnected by the meandered sections, is etched on the conducting plane and placed in the direction transverse to the $\mathrm{CPW}$ feedline. All the horizontal end-to-end slotline sections of dimensions $L \times W$ behave as the radiators or array elements, while the meandered slotline sections of length $l+2 t$ and uniform width $w$ serve as the feeding network. The radiating slotline sections take the form of resonant half-wavelength slot antennas rendering their lengths $L$ to equal approximately a half guided-wavelength $\lambda_{\mathrm{g}} / 2$ at the slotline resonance. Due to the in-line series-feed arrangement of the radiating elements in the proposed antenna, any radiating slotline section but the last and its next adjacent meandered section are required together to provide a phase delay of $360^{\circ}$ at resonance to ensure the in-phase excitation of all the radiating elements. In other words, their lengths should sum up to be a guided-wavelength at the slotline resonance, and thus gives the length of the meandered slotline sections $l+2 t=\lambda_{\mathrm{g}}-L \cong \lambda_{\mathrm{g}} / 2$.

The instantaneous electric field distribution on the apertures of the proposed antenna at resonance is shown in Fig. 2. The arrows indicate the directions and magnitudes of the electric 


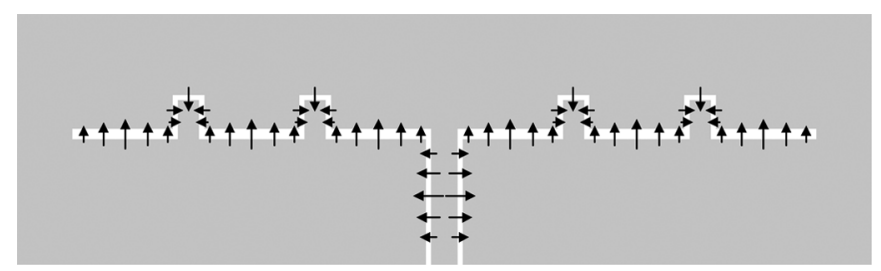

Fig. 2. Instantaneous electric field distribution at resonance for the antenna shown in Fig. 1.

fields. Note that the fields on the collinear radiating slotline sections are in the same phase and tend to reinforce the broadside radiation in the $\mathrm{H}-$ plane ( $\mathrm{x}-\mathrm{z}$ plane) pattern. However, the fields on the horizontal arms of the meandered slotline sections are in the opposite direction of the radiating slots and tend to partially cancel the reinforced broadside radiation. In order to reduce such cancellation, we properly shorten the length of the horizontal arms $l$. As $l<0.1 \lambda_{\mathrm{g}}$, the substantial mutual coupling effect between neighboring radiating slotline sections would greatly complicate the antenna design, which sets a lower limit for $l$.

Also note in Fig. 2 that the electric fields on the two closelyspaced vertical arms of the meandered slotline sections are in the opposite directions, thus their radiations will mostly cancel out in the far field region resulting in satisfactorily low crosspolarization levels in both principle planes. The E-plane (y-z plane) cross-polarization levels would be even lower because of the symmetry of the antenna structure along the $y$-axis and the CPW (even) mode operation of the feedline.

Judging from the above, the proposed in-line series-feed collinear slot array antenna fed by a CPW can be viewed as an equispaced linear array of parallel end-to-end half-wavelength slots with in-phase excitation. The antenna gain is thus increased by this simple array arrangement. We may note in passing that the shunt slotline pair of the proposed antenna discussed so far is terminated by short circuits as shown in Fig. 1. In the following subsections, its open-ended counterparts will be investigated.

\section{B. Open-Ended Case-Using Slotline Circular Open Stubs}

Based on the design guidelines and the operation principles illustrated by the short-ended case, we can similarly terminate the shunt slotline pair by open circuits. This subsection is devoted to investigating the in-line series-feed collinear slot array antenna terminated by the slotline circular open stubs, which have been extensively used as open circuits in the CPW transition design [14]. The configuration of this open-ended antenna is shown in Fig. 3, and the corresponding electric field distribution at resonance is illustrated in Fig. 4. The field distribution on the apertures except the circular open stubs remains the same as that in Fig. 2. The field distribution is kept unchanged simply because the length of the end slots is halved, which indicates $L_{\text {end }} \cong L / 2$ or $\lambda_{\mathrm{g}} / 4$ at the slotline resonance. Radiation patterns of the open-ended design are thus similar to those of the short-ended one. As for the open-circuit terminations, the diameter of the slotline circular open stubs $D$ is chosen to be approximately $\lambda_{\mathrm{g}} / 4$ at the slotline resonance. According to the boundary condition at the junctions of the end

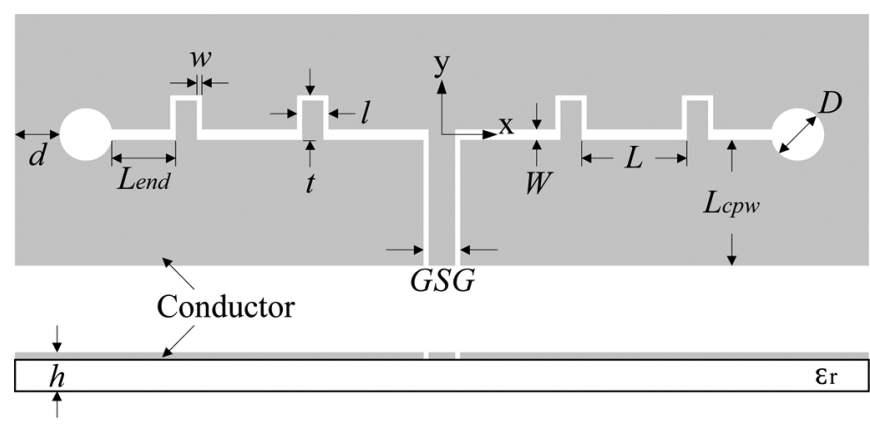

Fig. 3. Geometry of CPW-fed six-element in-line series-feed collinear slot array antenna terminated by slotline circular open stubs.

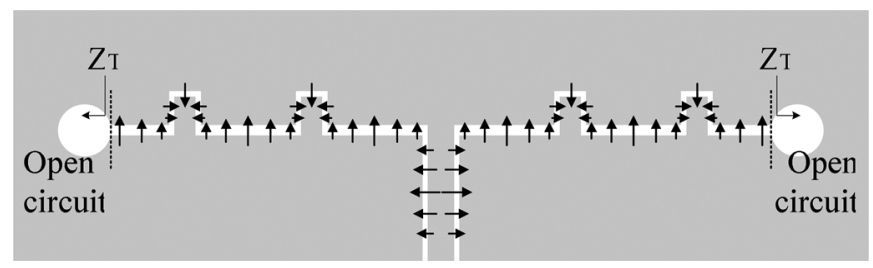

Fig. 4. Instantaneous electric field distribution at resonance for the antenna shown in Fig. 3.

slots and the circular open stubs, the shortened end slots behave as the resonant quarter-wavelength slot antennas in the proposed open-ended design. Note in Fig. 4 that the field distribution on the quarter-wavelength end slots not only adapts to the open-circuit terminations but also preserves the field distribution on the radiating half-wavelength slotline sections. It should be mentioned that the residual field on the circular open stubs, which is not shown in Fig. 4 for clarity, inevitably radiates to the far field and compensates the decrease in antenna gain caused by halving the end slots.

Since the only modification we have done to the open-ended design is halving the length of the end slots, the design considerations for all geometric parameters of the short-ended case discussed in the preceding subsection can also be applied to the open-ended one except for the end slots and the terminations.

It is worth mentioning that the above discussion still holds as the circular open stubs are replaced by other types of slotline open circuits, such as the radial open stubs [14] and the truncated open ends. The former is essentially the same as the slotline circular open stub, while the latter is far more compact in size. Therefore, in the next subsection, the compact open-ended design with the shunt slotline pair terminated by the truncated open ends will be studied.

\section{Compact Open-Ended Case-Using Truncated Ground Plane and Substrate}

The configuration of the compact open-ended in-line series-feed collinear slot array antenna fed by a CPW is depicted in Fig. 5. The shunt slotline pair is terminated by the truncated open ends, where both the ground plane and the dielectric substrate are truncated, and accordingly requires no extra ground plane and substrate area. The length of the end slots is also halved for preserving the field distribution. Therefore, the total width and antenna area of this open-ended design are reduced significantly. The field distribution on the shunt slotline pair 


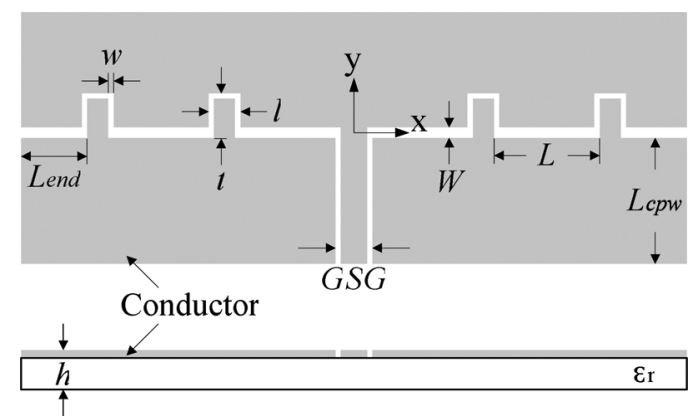

Fig. 5. Geometry of CPW-fed six-element in-line series-feed collinear slot array antenna terminated by truncated open ends.

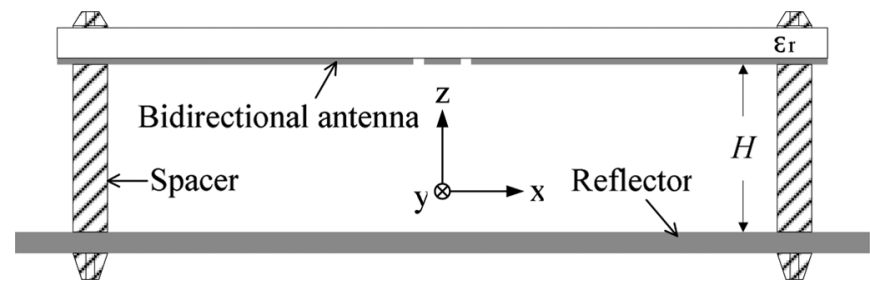

Fig. 6. Cross-section of the reflector-backed arrangement.

of this compact design is nearly the same as that shown in Fig. 4 and results in similar radiation patterns. However, due to the halved end slots and the directly-truncated open ends, the antenna gains are slightly lower than those of the preceding designs.

\section{Reflector-Backed Design for Unidirectional Radiation}

In many high-gain applications, antennas with unidirectional radiation patterns are preferable. Although the presented in-line series-feed collinear slot array antennas radiate bidirectionally, they can also provide unidirectional radiation with the aid of a reflector. The arrangement of the reflector-backed design for unidirectional radiation is illustrated in Fig. 6. An additional plane conductor sheet serving as a reflector is placed below the planar bidirectional antenna and parallel to the antenna surface. The reflector area is chosen to be slightly larger than the total substrate area of the bidirectional antenna for better back-lobe suppression. As indicated in Fig. 6, the bidirectional antenna is made bottom up since the slot antennas radiate more power to the side of the dielectric substrate than to the side of metallization for the electrically thin substrates and the semi-infinite substrate [15]. It may not be true for the electrically thick substrates, since a portion of the power radiated to the substrate side would leak away in the form of surface waves supported by the dielectric structure if the substrate is thick enough. Also, phase cancellation may occur at substrate thicknesses of near odd integer multiples of a quarter wavelength such that the front (substrate side)-to-back (metal side) ratio of the antenna is high. The front-to-back ratios for the bidirectional prototype antennas on the electrically thin substrate in this paper are in the range of 0.79 to $0.66 \mathrm{~dB}$. Furthermore, the optimal spacing $H$ between the bidirectional antenna and the reflector is a quarter free-space wavelength with respect to the beamwidth of the main beam, the front-to-back ratio, and the input impedance response according
TABLE I

GeOMETRIC PARAMETERS FOR SIX-ELEMENT PROTOTYPE ANTENNAS

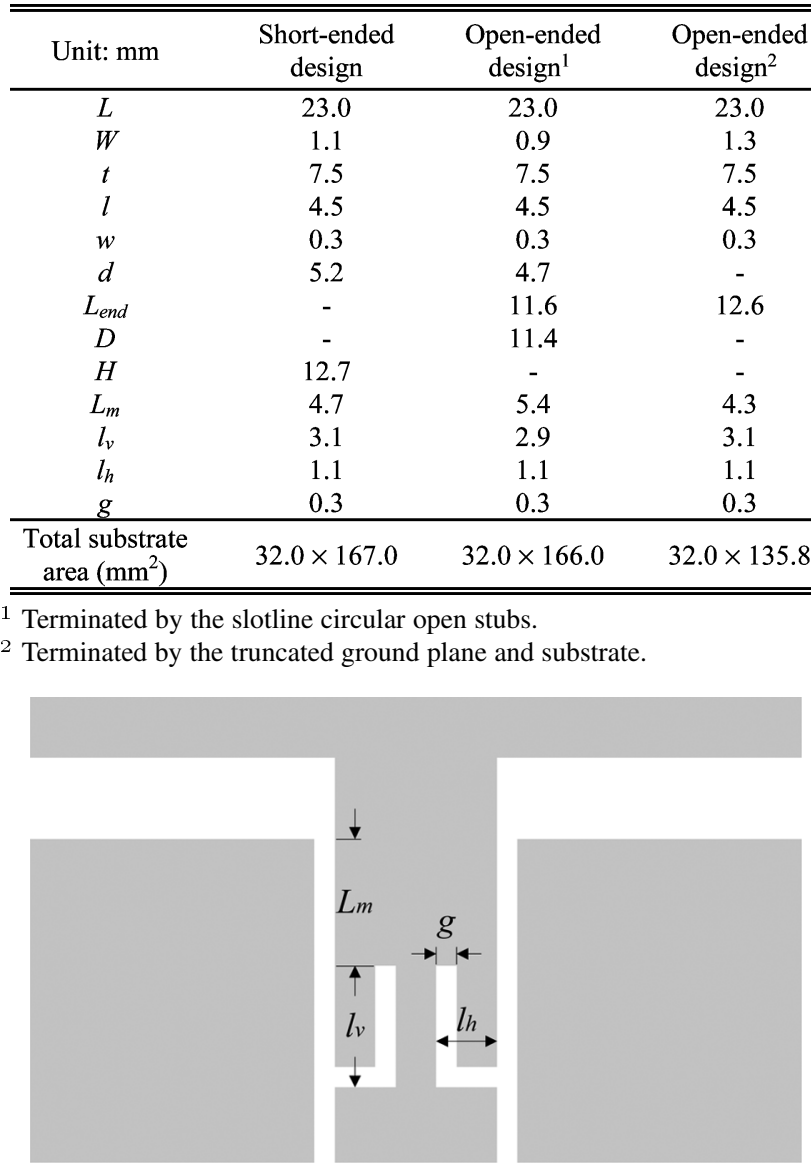

Fig. 7. Enlarged view of the CPW-to-slotlines T-junction with input matching circuit.

to [16]. The TEM mode between the ground plane and the reflector, which may cause additional power loss, is negligible in our reflector-backed design because both the ground plane and the reflector are of finite extent along the y-axis and the spacing between them is quite large. In addition to obtaining the unidirectional patterns, such a reflector-backed arrangement can also significantly increase the peak gain of the antenna. The mechanism for the gain improvement is that the power radiated into the side of metallization travels downward and is then reflected back by the reflector. The backside radiation is thus suppressed, and moreover, the frontward radiation is reinforced by the in-phase reflected one.

\section{EXPERIMENTAL RESULTS}

\section{A. Prototype Antennas and Input Matching Circuit}

In order to verify the performances of the proposed antennas, three prototype antennas, including one short-ended and the two open-ended in-line series-feed collinear slot array antennas all with six radiating elements, are implemented and tested. The reflector-backed arrangement is then applied to the short-ended prototype for comparison. Prototype antennas are designed at $5.5 \mathrm{GHz}$ following the fore-mentioned design guidelines. Throughout the design process, simulations are carried out on 


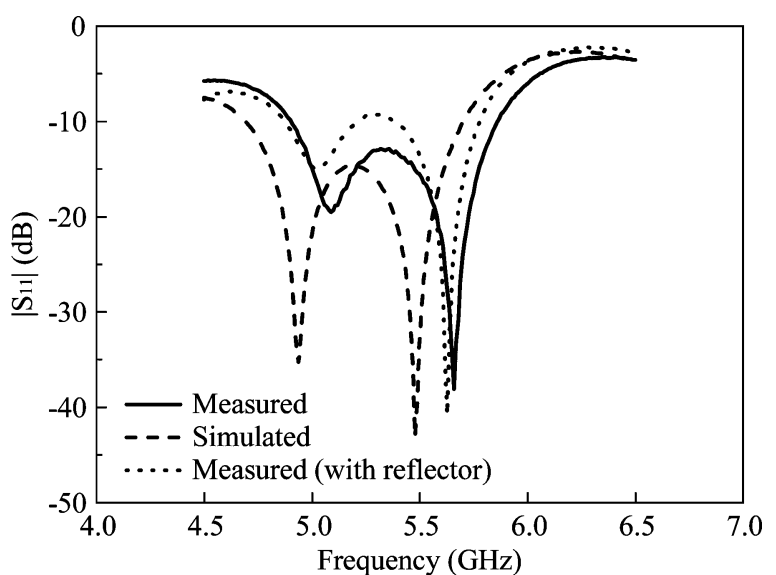

(a)

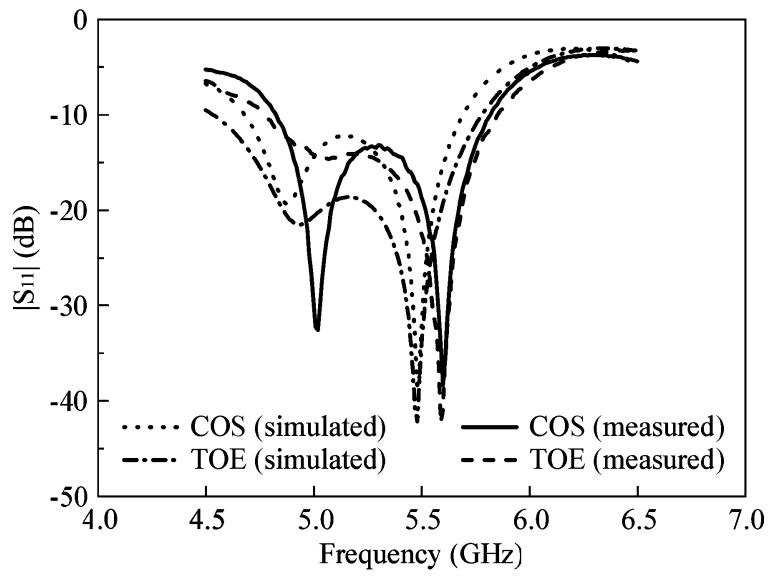

(b)

Fig. 8. Measured and simulated input return losses of (a) the short-ended prototype antenna and (b) the two open-ended prototype antennas. (COS: circular open stubs, TOE: truncated open ends).

the package software HFSS from Ansoft. All the prototype antennas are fabricated on the FR-4 substrate with dielectric constant $\varepsilon_{\mathrm{r}}=4.3$, loss tangent $\tan \delta=0.02$, and thickness $h=0.6 \mathrm{~mm}$. In these antennas, the $50 \Omega \mathrm{CPW}$ feedlines are of the same dimensions: $S=3.0 \mathrm{~mm}, G=0.3 \mathrm{~mm}$, and $L_{c p w}=15.5 \mathrm{~mm}$. The length of the radiating slotline sections $L$ is fixed at $23.0 \mathrm{~mm}$, which is slightly longer than a half guided-wavelength of the slotline at $5.5 \mathrm{GHz}$. The remaining parameters are listed in Table I. Note that we have four additional parameters, namely $L_{m}, l_{h}, l_{v}$, and $g$, due to the input matching circuit utilized in the prototype antennas. The enlarged view of the CPW-to-slotlines T-junction with the input matching circuit is depicted in Fig. 7. By properly adjusting $L_{m}$ and $l_{v}$, the required input impedance of $50 \Omega$ can be obtained at $5.5 \mathrm{GHz}$ for the prototype antennas. Table I lists the optimal values of $L_{m}$ and $l_{v}$ for the prototype antennas with $l_{h}$ and $g$ fixed.

\section{B. Input Return Loss Responses}

Measured and simulated input return losses of the short-ended prototype antenna without and with the reflector are plotted in Fig. 8(a), while those of the open-ended designs

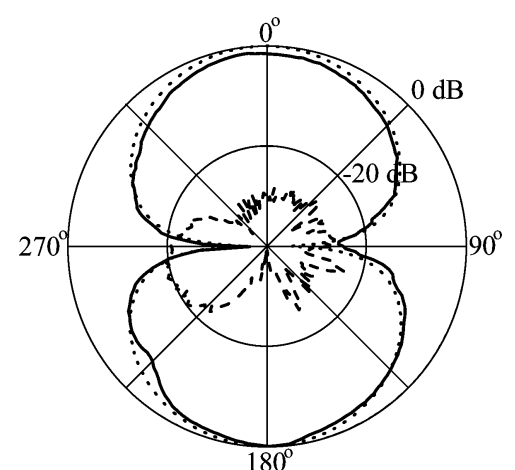

(a)

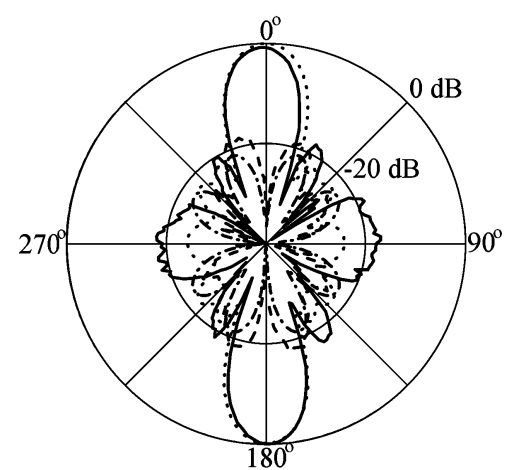

(b)

Fig. 9. Measured and simulated (a) E- and (b) H-plane patterns of the short-ended prototype antenna. Solid line: measured co-polarization, dashed line: measured cross-polarization, dotted line: simulated co-polarization, dash-dotted line: simulated cross-polarization.

terminated by the slotline circular open stubs and the truncated open ends are plotted in Fig. 8(b). The measured 10-dB return loss bandwidths of the four cases are $17.6 \%(4.91-5.86 \mathrm{GHz})$, $7.0 \%(5.39-5.78 \mathrm{GHz}), 18.6 \%(4.83-5.82 \mathrm{GHz})$, and $20.3 \%$ $(4.77-5.85 \mathrm{GHz})$, respectively. The measured and simulated responses are in good agreements except for the common frequency shift of about $0.1 \mathrm{GHz}$ between them. The response curves of the short-ended and the two open-ended designs exhibit excellent input match at the resonant frequency $(5.5 \mathrm{GHz}$ for simulation and $5.6 \mathrm{GHz}$ for measurement) and demonstrate the effectiveness of the matching circuit. Note in Fig. 8(a) that the reflector-backed arrangement inevitably degrades the input matching condition. However, the degradation remains negligible near $5.6 \mathrm{GHz}$.

\section{Radiation Performances}

E- and H-plane patterns of the six-element short-ended prototype antenna at the resonant frequency are plotted in Fig. 9(a) and (b), respectively. The radiation patterns are broadside bidirectional. Since the radiating elements are placed end-to-end along the x-axis, the E-plane pattern plotted in Fig. 9(a) is nearly the same as that of a slot dipole antenna. The proposed linear array structure well shapes the H-plane pattern as shown in Fig. 9(b). The half-power beamwidths of the main beams in Fig. 9(b) are about $15^{\circ}$ and $17^{\circ}$, and the side-lobe levels are lower than $-18 \mathrm{~dB}$. Also note that the cross-polarization levels in both principle planes are satisfactorily low. 


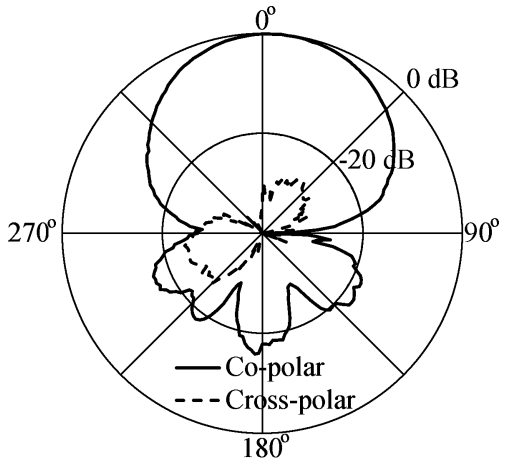

(a)

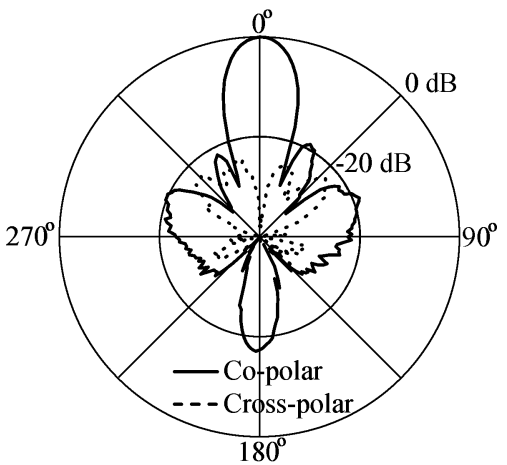

(b)

Fig. 10. (a) E- and (b) H-plane patterns measured at $5.6 \mathrm{GHz}$ for the shortended prototype antenna with reflector.

For comparison, the E- and H-plane patterns measured at $5.6 \mathrm{GHz}$ for the same antenna with the reflector-backed arrangement are plotted in Fig. 10(a) and (b), respectively. It can be seen that, with the back reflector, the frontward radiation patterns are only slightly changed, the half-power beamwidths of the frontward main beams in the E- and $\mathrm{H}$-planes are slightly narrowed from $69^{\circ}$ and $17^{\circ}$ to $61^{\circ}$ and $16.5^{\circ}$, respectively, while the backward radiations are effectively suppressed. The radiation patterns are thus unidirectional with the front-to-back ratio as high as $17 \mathrm{~dB}$, and the cross-polarization levels are even lower than those of Fig. 9(a) and (b).

Since the terminations of the shunt slotline pair mainly influence the field distribution along the $\mathrm{x}$-axis, the E-plane patterns of both the open-ended prototype antennas are nearly the same as that of the short-ended one, and hence they are omitted here. The H-plane patterns of the two open-ended designs with six radiating elements are plotted in Fig. 11(a) and (b), respectively. The similarity among the H-plane patterns shown in Fig. 11(a) and (b) and 9(b) can be easily observed. The reason, as mentioned earlier, is that the field distribution on the radiating slotline sections are kept unchanged, no matter what the short ends, the slotline circular open stubs, or the truncated open ends are used to terminate the shunt slotline pair. But the main differences among them are the higher side-lobe levels in Fig. 11(a) and (b) than in Fig. 9(b) due to the halved end slots in the open-ended designs.

The peak gains of the prototype antennas measured within their respective operating bands are compared and plotted in Fig. 12. All the gain responses are very stable. The in-band peak

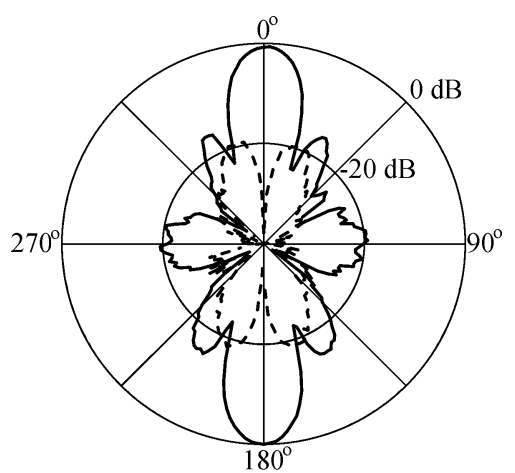

(a)

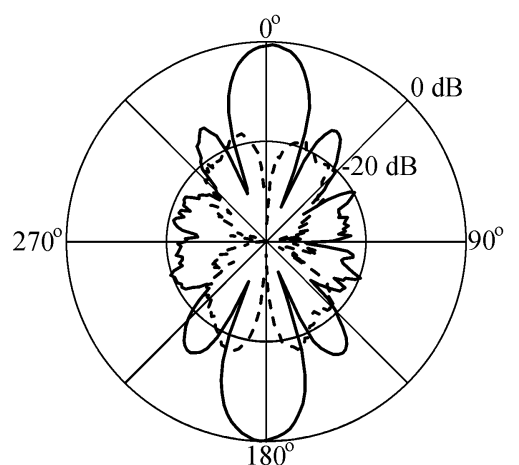

(b)

Fig. 11. H-plane patterns measured at $5.6 \mathrm{GHz}$ for the open-ended prototype antennas terminated by (a) slotline circular open stubs and (b) truncated open ends. Solid line: co-polarization, dashed line: cross-polarization.

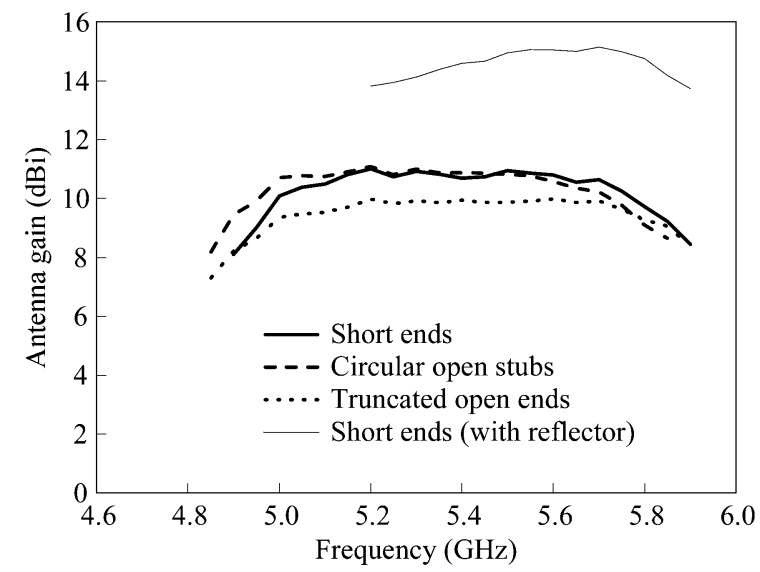

Fig. 12. Measured in-band peak gains of the prototype antennas.

gains of the prototype antennas with the short ends, the slotline circular open stubs, and the truncated open ends are in the ranges between 11.0 and $8.1 \mathrm{dBi}, 11.1$ and $8.2 \mathrm{dBi}$, and 10.0 and $7.3 \mathrm{dBi}$, respectively. The former two designs exhibit similar gain responses, both of which are approximately $1 \mathrm{~dB}$ higher than that of the latter one. The peak gains of the short-ended design are further increased by the reflector-backed arrangement, and fall in the range between 15.1 and $13.8 \mathrm{dBi}$. The efficiencies of the prototype antennas are listed as follows: $87.5 \%$ for the short-ended design, $86.2 \%$ for the open-ended design terminated by slotline circular open stubs, $87.2 \%$ for the open-ended design terminated by truncated open ends, and $88.4 \%$ for the short-ended design with reflector. These efficiencies are a few 
percents lower than those expected. The reason is mainly due to the dielectric loss existed in the substrate.

\section{CONCLUSION}

The in-line series-feed collinear slot array antenna fed by a CPW has been investigated. The shunt slotline pair of the antenna can be terminated by either short or open circuits. Merely by halving the length of the end slots, the open-ended design can provide radiation performances similar to those of the shortended one. A more compact design terminated by the truncated open ends has also been discussed and exhibited comparable performances. Although the presented antennas radiate bidirectionally, they can radiate unidirectional patterns with the aid of a reflector. In addition to the design flexibility, the simple array configuration, the compact size, and the uniplanar structure make the proposed antenna suitable for use in many wireless applications, such as the WLAN access points and the base stations of the fixed BWA systems.

\section{REFERENCES}

[1] [Online]. Available: http://www.grouper.ieee.org/groups/802/16

[2] X.-H. Yang and W.-X. Zhang, "Coplanar waveguide antenna arrays for MIC/MMIC at millimetre wave frequencies," Electron. Lett., vol. 26, no. 18, pp. 1464-1465, Aug. 1990.

[3] E. A. Soliman, S. Brebels, G. Vandenbosch, and E. Beyne, "X-band brick wall antenna fed by CPW," Electron. Lett., vol. 34, no. 9, pp. 836-838, Apr. 1998.

[4] H. Kobayashi and Y. Yasuoka, "Slot-array antennas fed by coplanar waveguide for millimeter-wave radiation," IEEE Trans. Microwave Theory Tech., vol. 46, no. 6, pp. 800-805, June 1998.

[5] S. Sierra-Garcia and J.-J. Laurin, "Study of a CPW inductively coupled slot antenna," IEEE Trans. Antennas Propag., vol. 47, no. 1, pp. 58-64, Jan. 1999.

[6] T.-F. Huang, S.-W. Lu, and P. Hsu, "Analysis and design of coplanar waveguide-fed slot antenna array," IEEE Trans. Antennas Propag., vol. 47, no. 10, pp. 1560-1565, Oct. 1999.
[7] M. Qiu, M. Simcoe, and G. V. Eleftheriades, "High-gain meanderless slot arrays on electrically thick substrates at millimeter-wave frequencies," IEEE Trans. Microwave Theory Tech., vol. 50, no. 2, pp. 517-528, Feb. 2002.

[8] A. Grbic and G. V. Eleftheriades, "Leaky CPW-based slot antenna arrays for millimeter-wave applications," IEEE Trans. Antennas Propag., vol. 50, no. 11, pp. 1494-1504, Nov. 2002.

[9] S.-Y. Chen and P. Hsu, "Open-ended rampart slot array antenna fed by a CPW," IEEE Antennas Wireless Propag. Lett., vol. 4, pp. 320-322, 2005.

[10] R. Johnson and H. Jasik, Eds., Antenna Engineering Handbook, 3rd ed. New York: McGraw-Hill, 1993, pp. 27-14-27-15.

[11] C. S. Franklin, "Improvements in wireless telegraph and telephone aerials," U.K. British Patent GB242342, Nov. 5, 1925.

[12] C. S. Franklin, "Wireless telegraph and telephone aerial," U.S. Patents 1821936 and 1914886, Sep. 8, 1931, and Jun. 20, 1933.

[13] C. S. Franklin and E. Green, "Antenna," U.S. Patent 1957949, May 8, 1934.

[14] R. N. Simons, Coplanar Waveguide Circuits, Components, and Systems. New York: Wiley-Interscience, 2001, pp. 319-334.

[15] M. Kominami, D. M. Pozar, and D. H. Schaubert, "Dipole and slot elements and arrays on semi-infinite substrates," IEEE Trans. Antennas Propag., vol. 33, no. 6, pp. 600-607, Jun. 1985.

[16] Y. Yoshimura, "A microstripline slot antenna," IEEE Trans. Microwave Theory Tech., vol. 20, no. 11, pp. 760-762, Nov. 1972.

Shih-Yuan Chen is with the Department of Electrical Engineering and the Graduate Institute of Communication Engineering, National Taiwan University, Taipei, Taiwan, R.O.C.

I-Ching Lan is with the Department of Electrical Engineering and the Graduate Institute of Communication Engineering, National Taiwan University, Taipei, Taiwan, R.O.C.

Powen Hsu is with the Department of Electrical Engineering and the Graduate Institute of Communication Engineering, National Taiwan University, Taipei, Taiwan, R.O.C 